\title{
Comparison of high resolution cytogenetics, fluorescence in situ hybridisation, and DNA studies to validate the diagnosis of Prader-Willi and Angelman's syndromes
}

\author{
A Smith, M Prasad, Z-M Deng, L Robson, $T$ Woodage, $R J$ Trent
}

\begin{abstract}
Eighty seven referrals with Prader-Willi syndrome and 49 with Angelman's syndrome were studied. High resolution cytogenetics was performed on all probands. Molecular studies, performed on the proband and both parents in each case, utilised multiple probes from within and distal to the 15(q11-13) region in order to establish the presence of DNA deletion or uniparental disomy. In addition, FISH, with probes at D15S11 and GABR 33 from the Prader-Willi syndrome/Angelman's syndrome region, was performed on a subset of 25 of these patients.
\end{abstract}

In the referral group with Prader-Willi syndrome, 62 patients had a normal karyotype and 25 were deleted on high resolution cytogenetics. Twenty nine were found to be deleted with DNA techniques. In the Angelman's syndrome group, 37 had a normal karyotype and 12 were deleted on high resolution cytogenetics while 26 were deleted on molecular studies. The diagnosis was reassessed in 35 referrals with PraderWilli syndrome and 11 with Angelman's syndrome following a non-deleted, nondisomic result. Of individuals who were neither deleted nor disomic on DNA studies, a false positive rate of $11.4 \%$ (4/35) for Prader-Willi syndrome and 16.7\% (2/12) for Angelman's syndrome was found for a cytogenetically detected deletion. The false negative rate for deletion detected on high resolution cytogenetics was $19 \cdot 5 \%$ (12/62) for PraderWilli syndrome and $35 \%(13 / 37)$ for Angelman's syndrome. Thus high resolution cytogenetics was shown to be unreliable for deletion detection and should not be used alone to diagnose either syndrome. There were no discrepancies with FISH in 25 cases when FISH was compared with the DNA results, indicating that FISH can be used reliably for deletion detection in both syndromes.

(Arch Dis Child 1995; 72: 397-402)

Keywords: deletion, uniparental disomy, abnormal phenotype.

The Prader-Willi syndrome was first described in 1956 in nine patients who had diminished fetal activity, severe infantile hypotonia, feeding problems in infancy, hypogonadism and hypogenitalism, short stature, small hands and feet, delayed psychomotor development, characteristic facial appearance, mild mental retardation, insatiable hunger leading to the onset of gross obesity during early childhood, behaviour problems, and tendency to develop diabetes in adolescence. ${ }^{1}$ These features can be quite variable between individual patients, and a diagnostic scoring system has been devised to aid diagnosis. ${ }^{2}$ The scoring system gives points to features present from birth to 3 years of age and for features after 3 years of age, when it is generally considered that the diagnosis is more straightforward. Angelman's syndrome was first described in 1965 on the basis of features in three young children. ${ }^{3}$ These features included severe mental retardation, epilepsy, tongue thrusting and drooling, an ataxic gait, and microcephaly. The facial features of Angelman's syndrome are subtle and nonspecific $^{4}$ and atypical cases have been described. ${ }^{5}$ A simple reliable diagnostic test would be extremely valuable for both syndromes in early childhood when the diagnosis is first suspected.

The association between an interstitial deletion of chromosome 15 and Prader-Willi syndrome was shown in $1981,^{6}$ and a similar interstitial deletion of the same region of chromosome 15 was shown in Angelman's syndrome in $1987 .{ }^{7}$ Cytogenetically detected interstitial deletions provided the first laboratory diagnostic test for these syndromes and considerable reliance came to be placed on the results of high resolution cytogenetics. Deletions of the proximal part of chromosome $15 \mathrm{q}$ were reported in about $60 \%$ of patients with Prader-Willi syndrome and a similar proportion of those with Angelman's syndrome with high resolution cytogenetic techniques. ${ }^{8}$ Approximately one third were apparently normal cytogenetically, and 3-5\% showed other chromosomal anomalies involving chromosome 15.910

The development of molecular probes for the Prader-Willi syndrome and Angelman's syndrome region of 15(q11-13) enabled alternate or complementary means for the detection of deletions in patients. ${ }^{10-12}$ Molecular studies not only demonstrated deletion, but also the parent of origin of the deletion. The deleted 15(q11-13) chromosome in patients with Prader-Willi syndrome always originates from the father ${ }^{11} 13$ while in Angelman's syndrome patients the deletion originates 
exclusively from the maternal chromosome 15. ${ }^{12}$ DNA studies further showed that some cases of Prader-Willi syndrome and Angelman's syndrome displayed uniparental disomy - the inheritance of two copies of a chromosome pair from one parent and none from the other parent. ${ }^{13} 15$ In Prader-Willi syndrome the uniparental disomy is maternal whereas in Angelman's syndrome the two chromosomes 15 are inherited from the father. Uniparental disomy occurs in approximately $25 \%$ of Prader-Willi syndrome and in only $2-3 \%$ of Angelman's syndrome. Cases with neither deletion nor disomy have also been found - in Prader-Willi syndrome they occurred rarely and were frequently due to misdiagnosis. ${ }^{16}$ Non-deleted, non-disomic cases comprise $25-30 \%$ of Angelman's syndrome and are a particular concern because recurrence occurs commonly within this group. ${ }^{17}$ When reports of the DNA studies became available ${ }^{1618}$ some discrepancies were noted between cytogenetic results and the DNA findings.

The technique of fluorescence in situ hybridisation (FISH) is a relatively new molecular cytogenetic technique in which specific DNA probes are applied directly to chromosome spreads on slides. ${ }^{1920}$ These slides can be used for routine analysis first with high resolution $G$ banding, or FISH can be applied to freshly prepared preparations. The technique is simpler than standard DNA studies and does not require specimens from the parents of the patient for deletion detection. FISH has proved to be highly accurate with many probes. ${ }^{19-21}$ FISH utilising probes from the Prader-Willi syndrome/Angelman's syndrome chromosome region has not been formally compared with DNA studies in these patients.

In the present study, 87 patients with the referral diagnosis of Prader-Willi syndrome and 49 patients referred with the diagnosis of Angelman's syndrome were examined with high resolution cytogenetics and detailed molecular analysis. Several patients whose diagnosis did not clearly fit either syndrome but who displayed some similar features were also included. Examination of such individuals is important for determining the range of clinical features that could potentially be associated with deletion or uniparental disomy of chromosome $15 .^{1622-24}$ In addition a cohort of 25 of these patients (seven with Prader-Willi syndrome and 18 with Angelman's syndrome) was examined by FISH. From the comparison of these findings, a strategy for the laboratory diagnosis of Prader-Willi syndrome and Angelman's syndrome can be proposed.

\section{Patients and methods PATIENTS}

Patients with a diagnosis or suspicion of Prader-Willi syndrome or Angelman's syndrome were referred by clinicians throughout Australia and New Zealand or the diagnosis was made by one of us (AS) in consultation with the referring doctor. The criteria used to establish the diagnosis of Prader-Willi syndrome were neonatal hypotonia, hyperphagia in early childhood with obesity, mild mental retardation, short stature, the characteristic facies seen in Prader-Willi syndrome, and hypogonadism. The clinical evaluation of many of these patients has been previously described (patients 1-3024; individual patients ${ }^{25} 26$ ). Subsequent patients were classified in the same way using standard diagnostic criteria. $^{2}$ For Angelman's syndrome the features used as guidelines were a large mouth, happy smiling disposition, jerky ataxic or wide based gait (if walking), severe mental retardation, no speech, epilepsy, abnormal electroencephalography, and inappropriate outbursts of laughter. ${ }^{4}$ Angelman's syndrome patients 1-10 have been described, ${ }^{23}$ and also an individual case, ${ }^{27}$ and subsequent patients were diagnosed in the same way. Patients who did not completely satisfy the criteria were considered to be atypical. When the results of DNA testing became known and were normal (that is neither deletion nor uniparental disomy was present) the clinician was asked to reassess the patient and possibly reconsider the diagnosis.

Of the 87 patients with Prader-Willi syndrome there were 52 males and 35 females; ages ranged from newborn to 39 years. Among the 49 patients with Angelman's syndrome there were 26 females and 23 males; ages ranged from 1.5 to 32 years.

\section{CYTOGENETICS}

Cytogenetic analysis was performed on all probands either in the Children's Hospital, Camperdown laboratory or in the state or New Zealand laboratory associated with the referring doctor. Peripheral blood lymphocytes were cultured using methods that increase the frequency of late prophase to early metaphase chromosomes. ${ }^{22} 28$ Karyotype analysis with GTG banding ( $G$ band chromosomes with trypsin and Giemsa) was performed to search for a chromosome 15 microdeletion. Resolution varied from the 650 to 850 band stage but when difficulties of interpretation were encountered, the cytogenetic results were considered as non-deleted. The parents of probands were not routinely tested by cytogenetic techniques.

\section{DNA ANALYSIS}

Molecular studies were performed on the patients and both parents in each case. High molecular weight DNA was prepared from peripheral blood specimens according to standard methods. Polymorphism analysis was performed with restriction fragment length polymorphisms, variable number of tandem repeats, and the polymerase chain reaction based simple sequence repeats with probes for loci from within the Prader-Willi syndrome/Angelman's syndrome chromosome region (D15S18, D15S9, D15S11, D15S13, D15S10, D15S113, D15S97, GABRß3, D15S12) and outside the region distally on 
Deleted
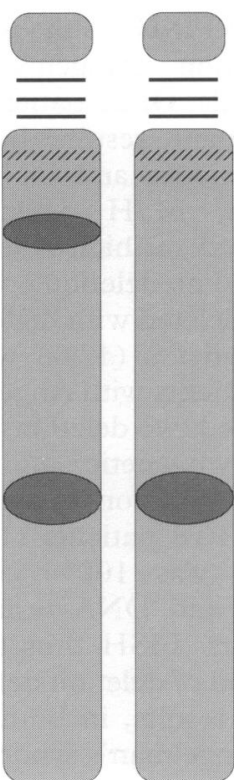

Figure 1 Representation of the expected signals on the two chromosomes 15 after FISH using either D15S11 or $G A B R \beta 3$ combined with the control probe at 15q22.

chromosome 15 (D15S24, ACTC, THBS1, D15S87, D15S86). DNA patterns at each polymorphic locus were recorded according to the bands seen on the autoradiographs or polyacrilamide gels. Detailed methods and the probe-enzyme combinations used have been previously described. ${ }^{22-24}$ Usually five polymorphisms per patient were sufficiently informative to establish whether a deletion or uniparental disomy was present, the parent of origin, or if the patient was non-deleted, nondisomic.

FISH

The probes used were from the 15(q11-13) region and comprised DNA specific for the loci D15S11 for Prader-Willi syndrome and GABR $\beta 3$ for Angelman's syndrome (Oncor). The probes are supplied as single stranded DNA labelled with digoxigenin. After hybridisation on to denatured chromosomal preparations ${ }^{19} 26$ detection was via immunocytochemical incubations with an antidigoxigenin mouse monoclonal antibody (Boehringer Mannheim) and a fluorescein conjugated sheep antimouse IgG antibody (Boehringer Mannheim). The preparation was counterstained with a combination of propidium iodide (Calbiochem) and 4',6-diamidino-2-phenylindole (Sigma). The probes available for FISH have been modified to provide ease of usage by the incorporation of a DNA marker (also digoxigenin labelled) on the long arm of chromosome 15 at q22 (fig 1). The distal marker acts as an internal control for successful hybridisation and aids in the quick assessment of a deletion (fig 2).

Results

The results of high resolution cytogenetics and
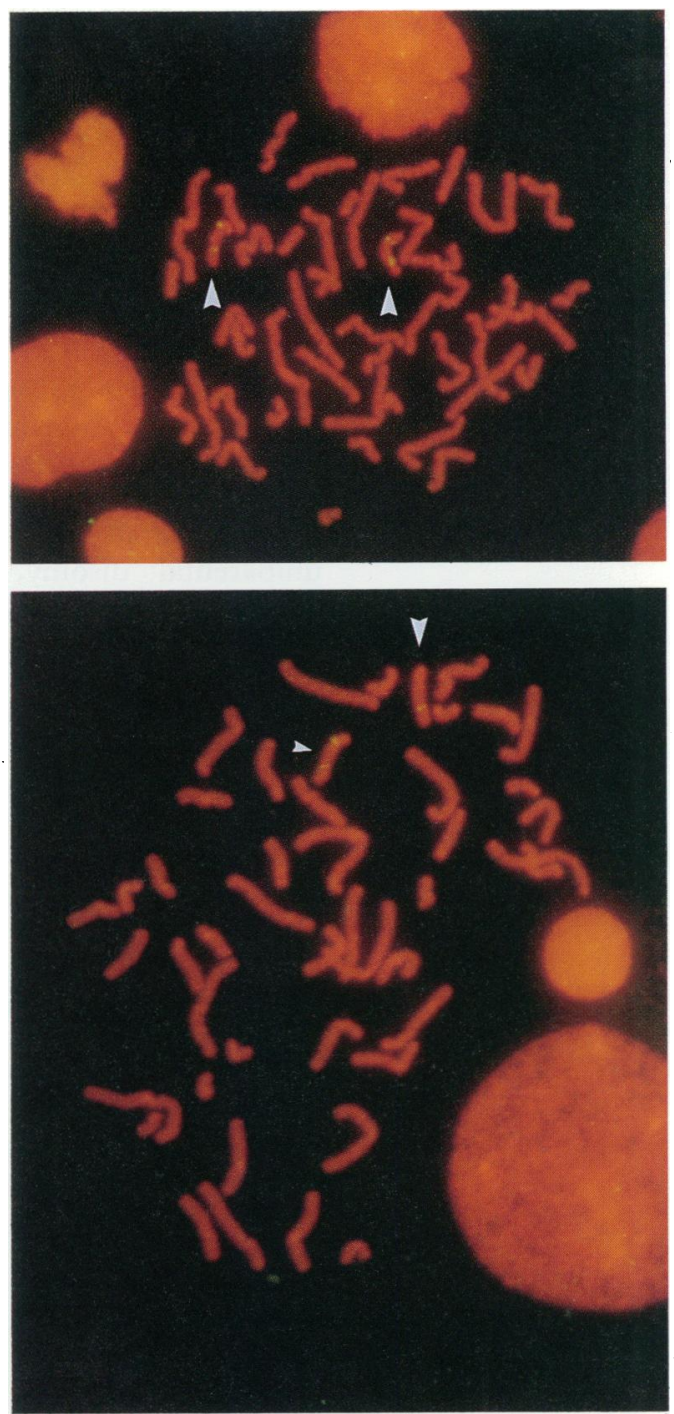

Figure 2 Upper: patient with Angelman's syndrome (number 17 in table 2) showing metaphase spread with two chromosomes 15 (arrowed) each containing two signals: one for the Angelman's syndrome region (recognised by probe $G A B R \beta 3$ ) and one from the $15 q 22$ region. The patient was non-deleted for GABR 33 . Lower: patient with Prader-Willi syndrome (number 1 in table 2) showing metaphase spread with two chromosomes 15. One chromosome has two signals (small arrowhead) and the other has a single signal (large arrowhead) emanating from the 15(q22) marker. The patient was deleted for D15S11.

DNA testing and their correlation are shown in table 1 . Of the total 87 patients referred with Prader-Willi syndrome, 62 showed normal chromosomes and 25 showed a deletion on high resolution cytogenetics. The DNA findings showed overall that 29 patients had a deletion, 20 had uniparental disomy, and 38 were non-deleted, non-disomic. All patients with deletion and uniparental disomy had classical Prader-Willi syndrome. Clinical reassessment of the 38 non-deleted, non-disomic patients resulted in 35 being reclassified as atypical Prader-Willi syndrome or non-Prader-Willi syndrome. Re-evaluation of the remaining three patients (case numbers 28, 29, 30 in Woodage et $a l^{24}$ ) indicated that they had features supportive of a diagnosis of PraderWilli syndrome and have remained in the classification of classical Prader-Willi syndrome patients. The 29 deleted patients and the 20 patients with uniparental disomy all had 
Table 1 Correlation of high resolution cytogenetic findings with the DNA results for all referrals

\begin{tabular}{|c|c|c|c|c|c|}
\hline & \multicolumn{2}{|c|}{ Cytogenetics } & \multicolumn{3}{|l|}{$D N A$} \\
\hline & Normal & Deleted & Deleted & $\begin{array}{l}\text { Uniparental } \\
\text { disomy }\end{array}$ & $\begin{array}{l}\text { Non-deleted, } \\
\text { non-disomic }\end{array}$ \\
\hline $\begin{array}{l}\text { Prader-Willi syndrome } \\
(\mathrm{n}=87) \\
\text { Angelman's syndrome } \\
(\mathrm{n}=49)\end{array}$ & $\begin{array}{r}62 \\
-\overline{37} \\
-\end{array}$ & $\begin{array}{l}2 \overline{5} \\
\overline{12}\end{array}$ & $\begin{array}{l}12 \\
17 \\
13 \\
10\end{array}$ & $\begin{array}{r}16 \\
4 \\
1 \\
1\end{array}$ & $\begin{array}{r}34 \\
4 \\
23 \\
1\end{array}$ \\
\hline
\end{tabular}

classical Prader-Willi syndrome. Among the 49 patients with Angelman's syndrome, 37 had normal chromosomes on high resolution cytogenetics, 12 had a deletion. The DNA findings showed overall that 23 were deleted, two had uniparental disomy, and 23 had normal chromosomes. Among these non-deleted, nondisomic patients with Angelman's syndrome, clinical reassessment indicated that 12 had classical features of Angelman's syndrome and have retained that diagnosis. The other 11 patients remain under review with monitoring of further clinical progress.

There was overall a low pick up rate of cytogenetic deletion (25 patients of 87 referrals or $29 \%$ in the Prader-Willi syndrome group and 12 in 49 or $25 \%$ in the Angelman's syndrome group). The four Prader-Willi syndrome patients with uniparental disomy and the four non-deleted, non-disomic patients $(8 / 25)$ represent a $32 \%$ false positive rate for the detection of a deletion by high resolution cytogenetics in Prader-Willi syndrome and $2 / 12(16 \cdot 6 \%)$ in Angelman's syndrome. The overall false negative rate for the Prader-Willi syndrome referrals $(12 / 62)$ is approximately $20 \%$ and for Angelman's syndrome is $13 / 37$ or $35 \%$. When only the 52 classical patients are considered the deletion detection rate for Prader-Willi syndrome becomes $48 \%$. High resolution cytogenetics cannot detect uniparental disomy so that if the 20 patients with uniparental disomy are excluded high resolution cytogenetics then detected deletion in

Table 2 Comparison of laboratory results on 25 patients with Prader-Willi syndrome (PWS) or Angelman's syndrome (AS)

\begin{tabular}{llllll}
\hline $\begin{array}{l}\text { Patient } \\
\text { No }\end{array}$ & $\begin{array}{l}\text { Sex/age } \\
\text { (years) }\end{array}$ & Diagnosis & $\begin{array}{l}\text { High resolution } \\
\text { cytogenetics }\end{array}$ & FISH & DNA \\
\hline 1 & M/2 & PWS & Deleted & Deleted & Deleted \\
2 & M/16 & PWS & ND & ND & NDND \\
$3^{\star}$ & F/15 & PWS & Deleted & ND & Uniparental disomy \\
$4^{\star}$ & M/5 & PWS & Deleted & ND & Uniparental disomy \\
5 & F/8 & PWS & Deleted & Deleted & Deleted \\
$6^{\star}$ & M/17 & PWS & ND & Deleted & Deleted \\
7 & M/15 & PWS & ND & ND & Uniparental disomy \\
8 & F/7 & AS & Deleted & Deleted & Deleted \\
9 & F/9 & AS & Deleted & Deleted & Deleted \\
10 & M/3 & AS & ND & ND & NDND \\
11 & F/2 & AS & ND & ND & NDND \\
$12^{\star}$ & F/11 & AS & ND & Deleted & Deleted \\
13 & M/10 & AS & ND & ND & NDND \\
14 & F/18 & AS & Deleted & Deleted & Deleted \\
$15^{\star}$ & M/18 & AS & ND & Deleted & Deleted \\
16 & M/12 & AS & Deleted & Deleted & Deleted \\
17 & F/4 & AS & ND & ND & NDND \\
18 & F/6 & AS & Deleted & Deleted & Deleted \\
19 & M/5 & AS & ND & ND & NDND \\
20 & F/4 & AS & ND & ND & NDND \\
21 & M/16 & AS & Deleted & Deleted & Deleted \\
$22^{\star}$ & M/18 & AS & Deleted & ND & Uniparental disomy \\
23 & F/4 & AS & ND & ND & NDND \\
24 & F/4 & AS & ND & ND & NDND \\
25 & F/3 & AS & ND & ND & NDND \\
& & & & & \\
\hline
\end{tabular}

NDND = non-deleted, non-disomic; $\mathrm{ND}=$ non-deleted.

*Indicates cases with discrepancy between high resolution cytogenetics and DNA results. There were no discrepancies between the FISH and DNA results.
25/32 classical Prader-Willi syndrome patients $(78 \%)$.

The FISH results were correlated with high resolution cytogenetics and DNA studies (table 2). Of 25 patients, there were six $(24 \%)$ discrepant results between high resolution cytogenetics and DNA. In Prader-Willi syndrome, FISH detected one deletion not detected on high resolution cytogenetics and showed no deletion on two patients considered to be deleted with high resolution cytogenetics; $3 / 7$ patients $(42 \%)$ were discrepant. Among the patients with Angelman's syndrome, FISH detected two deletions not seen by high resolution cytogenetics and could not confirm the high resolution cytogenetics deletion in one case; $3 / 18$ patients $(16 \cdot 6 \%)$ were discrepant. There was $100 \%$ correlation between the FISH and DNA results in this group of 25 patients. FISH thus appears to be a reliable method of deletion detection, validated against DNA results, in both Prader-Willi syndrome and Angelman's syndrome.

\section{Discussion}

The results of this study show considerable discrepancy between high resolution cytogenetics and DNA findings. Using DNA as the diagnostic standard, we found for Prader-Willi syndrome and Angelman's syndrome false positive rates of $12 \%$ and $17 \%$ and false negative rates of $20 \%$ and $35 \%$ respectively for deletion detection with high resolution cytogenetics. Overall, among the whole group of referrals with Prader-Willi syndrome, deletions were detected less frequently than expected, ${ }^{816}$ reflecting the finding that nearly half the patients were atypical. Among the classical Prader-Willi syndrome patients, there were many with uniparental disomy (38\%) and high resolution cytogenetics cannot pick up this group. Thus it is clear that reliance cannot be placed upon the result of the high resolution cytogenetics test for the diagnosis of PraderWilli syndrome or Angelman's syndrome. Discrepant results and submicroscopic DNA deletions not detected on high resolution cytogenetics have been reported in both PraderWilli syndrome and Angelman's syndrome in smaller groups of patients. ${ }^{161829}$

Reasons for discrepancies between high resolution cytogenetics and DNA studies have been documented. False negative results are partly due to the greater resolution of DNA compared with cytogenetics. At the 1000 band stage of resolution, one cytogenetic band could contain five megabases of DNA, so that a normal high resolution cytogenetics result does not exclude a smaller DNA deletion. False positive results can be accounted for in part by common polymorphisms of the chromosome 15 pericentromeric region, which include duplications and deletions. ${ }^{18}$ 30-32 Replication asynchrony between homologues in the proximal long arm region has been demonstrated and can result in one chromosome 15 homologue appearing to be shorter than the other. ${ }^{33}$ It could be expected that the success of high resolution cytogenetics would vary 


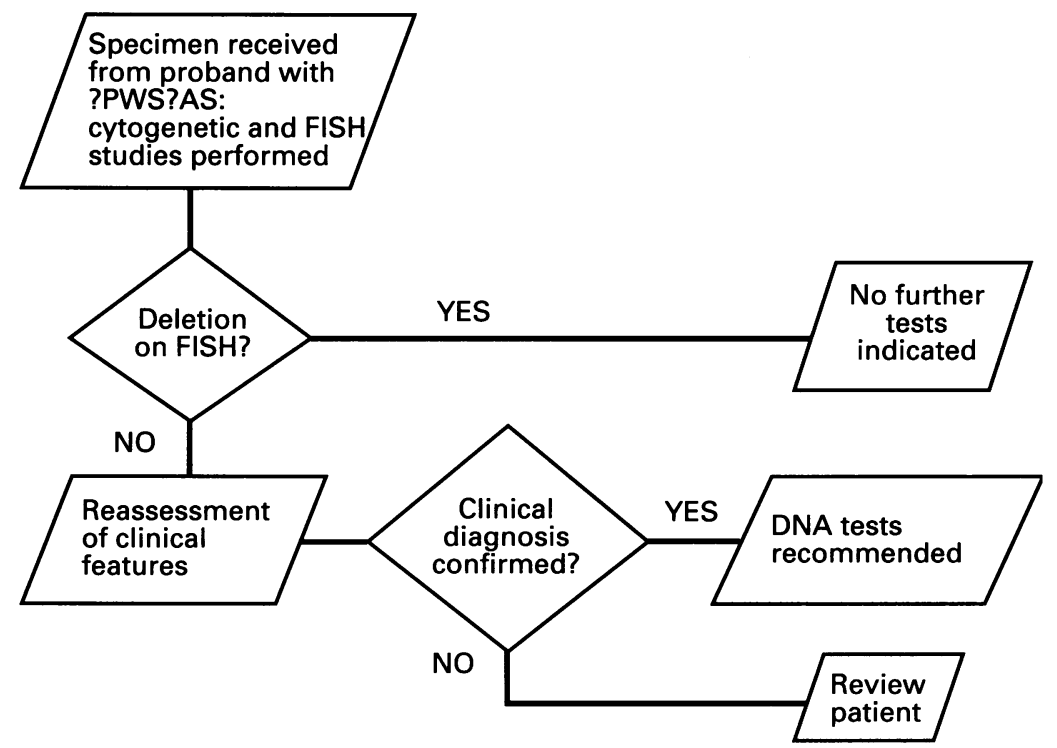

Figure 3 Flow diagram with suggested protocol for the investigation of Prader-Willi syndrome (PWS) and Angelman's syndrome (AS). the possibility of structural rearrangements involving chromosome 15 which occur in about 5\% of Prader-Willi syndrome and Angelman's syndrome individuals. For this reason, FISH cannot be the sole technique without cytogenetics, as has been suggested. ${ }^{37}$

A sensible strategy for the investigation of suspected Prader-Willi syndrome/Angelman's syndrome patients is outlined in fig 3 . Routine cytogenetics is performed first and then FISH on the same culture or slides as was used for the initial routine cytogenetics. Our procedure now is to make an extra slide for FISH at the time of the cytogenetic harvest. This protocol will diagnose the majority of both Prader-Willi syndrome and Angelman's syndrome patients as approximately $70 \%$ have a large DNA deletion. FISH accurately reflects the DNA deletion so that when a deletion is detected, no further DNA testing needs to be performed. In those cases in which a deletion is not detected by FISH, clinical reassessment is valuable to suggest if any further testing is warranted. Some cases may be withdrawn at this stage. If DNA testing is to be undertaken, this may follow standard polymorphic marker analysis, requiring specimens from the parents. For classical Prader-Willi syndrome patients, uniparental disomy will be shown to be the mechanism in the great majority of the non-deleted patients, 89 and verification of uniparental disomy can be sought by DNA testing with terminal 15q markers only. Alternatively, DNA testing could be performed by the methylation technique which relies on methylation sensitive restriction enzymes differentially cutting maternal and paternal DNA. ${ }^{38-40}$ The methylation patterns obtained can diagnose Prader-Willi syndrome and Angelman's syndrome accurately ${ }^{38-41}$ but cannot distinguish between the mechanism of deletion or uniparental disomy. ${ }^{38-41}$ Methylation testing is more rapid and less expensive than full polymorphic studies and does not require parental blood samples. Several methylation sites are present in the 15(q11-13) region, including $\mathrm{PW} 71^{38}$ which has proved to be useful for clinical diagnosis ${ }^{41}$ and can act as a back up when parental samples are not available. Among patients with Angelman's syndrome approximately one third remain unresolved with the current DNA probes available. It is important to establish the mechanism because the recurrence risks are very low for uniparental disomy but up to $50 \%$ if the patient is non-deleted, non-disomic. These patients should be kept under review for retesting as the Angelman's syndrome gene is defined.

While DNA testing can be time consuming and expensive, FISH has reduced by two thirds the number of cases requiring full DNA work up and is thus additionally cost effective. The selective use of DNA studies proposed with this strategy will keep the total cost of investigation to a minimum but also identify those patients where further detailed DNA studies are warranted.

We thank Professor D Sillence, Head, Department of Genetics, Children's Hospital, Camperdown, for his support. The DNA work was performed under a grant from the National Health and Medical Research Council, Australia. technique should replace high resolution cytogenetics in the diagnosis of these patients. Routine cytogenetics with GTG banding mus be performed on all suspected patients due to 
1 Prader A, Labhart A, Willi H. Ein Syndrom von Adipositas, Kleinwuchs, Kryptochismus und Oligophrenic nach myatonieartigem Zustand im Neuegebornenalter. Schweiz Med Wochenschr 1956; 86: 1260-1.

2 Holm VA, Cassidy SB, Butler MG, et al. Prader-Will syndrome: consensus diagnostic criteria. Pediatrics 1993, 91: 398-402.

3 Angelman HA. 'Puppet' children. A report on three cases. Dev Med Child Neurol 1965; 7: 681-8.

4 Clayton-Smith J, Pembrey ME. Angelman syndrome. f Med Genet 1992; 29: 412-5.

5 Clayton-Smith J, Webb T, Cheng XJ, Pembrey ME Malcolm S. Duplication of chromosome 15 in the region 15q11-13 in a patient with developmental delay and $15 \mathrm{q} 11-13$ in a patient with developmental delay and ataxia with similarities

6 Ledbetter DH, Riccardi VM, Airhart SD, Strobel RJ Keenan BS, Crawford JD. Deletion of chromosome 15 as a cause of the Prader-Willi syndrome. N Engl F Med 1981; 304: 325-9.

7 Magenis RE, Brown MG, Lacy DA, Budden S, LaFranch $S$. Is Angelman syndrome an alternate result of $\operatorname{del}(15)($ 1 1 1-13)? Am F Med Genet 1987; 28: 829-38.

8 Nicholls RD. Genomic imprinting and uniparental disomy in Angelman and Prader-Willi syndromes: a review. Am 7 Med Genet 1993; 46: 16-25.

9 Nicholls RD. Genomic imprinting and candidate genes in the Prader-Willi and Angelman syndromes. Curr Opin Genet Dev 1993; 3: 445-56.

10 Chan C-TJ, Clayton-Smith J, Cheng X-J, et al. Molecular mechanisms in Angelman syndrome: a survey of 93 patients. 7 Med Genet 1993; 30: 895-902.

11 Butler MG. Prader-Willi syndrome: current understanding of cause and diagnosis. Am 7 Med Genet 1990; 35: 319-32

12 Knoll JH, Nicholls RD, Magenis RE, Graham JM, Lalande M, Latt SA. Angelman and Prader-Willi syndromes share common chromosome 15 deletion but differ in parental origin of the deletion. Am 7 Med Genet 1989; 32: 285-90.

13 Nicholls RD, Knoll JH, Butler MG, Karam S, Lalande M Genetic imprinting suggested by maternal uniparetna heterodisomy in non-deletion Prader-Willi syndrome. Nature 1989; 342: 281-5.

14 Williams CA, Zori RT, Stone JW, Gray BA, Cantu ES, Ostrer H. Maternal origin of 15q11-13 deletions in Angelman syndrome suggests a role for genomic imprinting. Am 7 Med Genet 1990; 35: 350-3.

15 Malcolm S, Clayton-Smith J, Nichols M, Robb S Uniparental paternal disomy in Angelman syndrome. Uniparental paternal

16 Robinson WP, Bottani A, Yagang X, et al. Molecular, cytogenetic, and clinical investigations of Prader-
Willi syndrome patients. Am ₹ Hum Genet 1991; 49: 1219-34.

17 Wagstaff J, Shugart YY, Lalande $M$. Linkage analysis in familial Angelman syndrome. Am f Hum Genet 1993; 53 105-12.

18 Zackowski JL, Nicholls RD, Gray BA, et al. Cytogenetic and molecular analysis in Angelman syndrome. $\mathrm{Am} \mathrm{f} \mathrm{Med}$ Genet 1992; 46: 2-6.

19 Cremer T, Lichter P, Borden J, Ward DC, Manuelidis L. Detection of chromosome aberrations in metaphase and interphase tumor cells by in situ hybridisation using 235-46.

20 Trask BJ. Fluoresence in situ hybridisation: application in cytogenetics and gene mapping. Trends Genet 1991; 78 $149-54$

21 Gingrich JC, Shadravan F, Lowry SR. A fluorescence in situ map of human chromosome 21 consisting of 20 genetic and physical markers on the chromosome: localisation of 137 additional YAC and cosmid clones with respect to this map. Genomics 1993; 17: 98-105.
22 Trent RJ, Volpato F, Smith A, et al. Molecular and cytogenetic studies of the Prader-Willi syndrome. $\mathcal{F}$ Med Genet 1991; 28: 649-54

23 Deng Z-M, Woodage TJ, Smart R, Smith A, Trent RJ Novel patterns of inheritance of genetic disease are illustrated by the Angelman syndrome. Med f Aust 1993; 158: 813-6.

24 Woodage T, Deng Z-M, Prasad M, et al. A variety of genetic mechanisms are associated with the Prader-Willi syndrome. Am 7 Med Genet 1994; 54: 219-26.

25 Smith A, Noel M. A girl with the Prader-Willi syndrome and robertsonian translocation $45, \mathrm{XX}, \mathrm{t}(14 ; 15)(\mathrm{p} 11 \mathrm{q} 11)$ which was present in three normal family members. Hum which was present in thre

26 Smith A, Robson L, Neumann A, et al. Fluoresence in situ hybridisation and molecular studies used in the characterisation of a robertsonian translocation $(13 \mathrm{q} 15 \mathrm{q})$ in the Prater-Willi syndrome. Clin Genet 1993; 43: 5-8.

27 Smith A, Deng Z-M, Beran R, Woodage T, Trent RJ Familial unbalanced translocation $t(8 ; 15)(\mathrm{p} 23.3 ; \mathrm{q} 11)$ with uniparental disomy in Angelman syndrome. Hum Genet 1994; 93: 471-3.

28 Yunis JJ. High resolution of human chromosomes. Science 1976; 191: 1268-70.

29 Lai L-W, Erickson RP, Cassidy S. Clinical correlates of chromosome 15 deletions and maternal disomy in PraderWilli syndrome. Am f Dis Child 1993; 147: 1217-23.

30 Hood OJ, Rouse BM, Lockhart LH, Bodensteiner JB. Proximal duplications of chromosome 15: clinical dilemmas. Clin Genet 1986; 29: 234-40.

31 Hoo JJ, Chao MC, Samuel IP, Morgan AM. Proximal 15q variant as possible pitfall in the cytogenetic diagnosis of Prader-Willi syndrome. Clin Genet 1990; 37: 161-6.

32 Brookwell R, Veleba A. Proximal 15q variant with norma phenotype in three unrelated individuals. Clin Genet 1987, 31: $311-4$

33 Izumikawa Y, Naritomi K, Hirayama K. Replication asynchrony between homologs 15q11.2: cytogenetic evidence for genomic imprinting. Hum Genet 1991; 87: 1-5.

34 Ronne M. Double synchronisation of human lymphocyte cultures: selection for high resolution banded metaphase in the first and second division. Cytogenet Cell Genet 1985; 39: $292-5$.

35 Ledbetter DH, Cassidy SB. The etiology of Prader-Willi syndrome: clinical implications of the chromosome 15 abnormalities. In: Caldwell ML, Taylor RL, eds. Prader-Willi syndrome. New York: Springer-Verlag, 1988: 14-9.

36 Delach JA, Rosengren SA, Kaplan L, Greenstein RM, Cassidy SB, Benn PA. Comparison of high resolution tion (FISH) for the laboratory evaluation of Prader-Willi tion (FISH) for the laboratory evaluation of Prader-Will and Ange

37 Donaldson MDC, Chu CE, Cooke A, Wilson A, Green SA Stephenson JBP. The Prader-Willi syndrome. Arch Dis Child 1994; 70: 58-63.

38 Dittrich B, Robinson WP, Knoblauch H, et al. Molecula diagnosis of the Prader-Willi and Angelman syndromes by detection of parent-of-origin specific DNA methylation in 15q11-13. Hum Genet 1992; 90: 313-5.

39 Lerer I, Meiner V, Pashut-Lavon I, Abeliovich D. Molecular diagnosis of Prader-Willi syndrome. Parent of origin dependent methylation sites and non-isotopic detection of $(\mathrm{CA})_{n}$ dinucleotide repeat polymorphisms. Am fMed Genet 1994; 52: 79-84

40 Sutcliffe JS, Nakao M, Christian S, et al. Deletions of differentially methylated CpG island at the SNRPN gene define a putative impris

41 Chu CE, Cooke A, Shephenson JBP, et al. Diagnosis in Prader-Willi syndrome. Arch Dis Child 1994; 71: 441-2. 